\title{
Primary high-grade calcitonin-negative neuroendocrine carcinoma of the thyroid: a very rare cancer
}

\author{
Joseph A Chorny', John J Orrego² and José Manuel Cameselle-Teijeiro ${ }^{3}$ \\ 1Department of Pathology, ${ }^{2}$ Department of Endocrinology and Metabolism, Colorado Permanente Medical Group, \\ Denver, Colorado, USA, and ${ }^{3}$ Department of Anatomic Pathology, Clinical University Hospital, Santiago de \\ Compostela, Spain
}

\author{
Correspondence \\ should be addressed \\ to J A Chorny \\ Email \\ joseph.a.chorny@kp.org
}

\section{Summary \\ Most medullary thyroid carcinomas (MTCs) are low grade and produce calcitonin. There are some calcitonin-negative MTCs that produce only calcitonin gene-related peptide (CGRP). Rarely, MTCs are negative for calcitonin and CGRP peptides, but contain their corresponding mRNAs. Primary thyroid neuroendocrine neoplasms other than MTCs are extremely rare. We describe a primary high-grade neuroendocrine carcinoma that was negative for CGRP and calcitonin at both the protein and mRNA levels. A 42-year-old woman presented with a rapidly enlarging thyroid mass replacing most of the left lobe and isthmus. A computed tomography-guided core-needle biopsy was performed. The tumor was composed of sheets of small-to-medium sized epithelial cells. The cells were immunoreactive for pancytokeratin, synaptophysin, CD56 and thyroid transcription factor-1, but negative for CK7, CK20, CD45, CD99, ERG, chromogranin A, thyroglobulin, calcitonin, CGRP and carcinoembryonic antigen. The Ki-67 proliferation index was $~ 90 \%$. In situ hybridization was negative for calcitonin mRNA. The patient was initially diagnosed as having a small cell carcinoma. She was treated with cisplatin and etoposide (VP16), followed by radiation therapy. Given the excellent clinical course, the tumor was reviewed and reclassified as a high-grade neuroendocrine carcinoma (non-small-cell type). Heretofore, only a few other similar high-grade neuroendocrine tumors with negative markers of C-cell derivation have been reported. In our case, the patient is cancer free five years after diagnosis, but in the other cases, the outcome was poor.}

\section{Learning points:}

- There are neuroendocrine carcinomas of the thyroid that do not produce calcitonin or calcitonin gene-related peptide.

- This category of calcitonin-negative neuroendocrine carcinomas is heterogeneous, consisting of low- and highgrade tumors.

- The high-grade neuroendocrine carcinomas of the thyroid are rare and generally have a poor prognosis. They are divided into small cell and non-small cell or large cell types.

\section{Background}

Medullary thyroid carcinomas (MTCs) are usually immunoreactive for calcitonin. Some calcitonin-negative MTCs express only calcitonin gene-related peptide
(CGRP), and rare MTCs are negative for calcitonin and GCRP peptides, but may show positivity for their corresponding mRNAs. Primary thyroid neuroendocrine 
neoplasms other than MTCs are rare. We describe a case of a calcitonin-negative high-grade neuroendocrine carcinoma of the thyroid gland to provide our experience and management with this very rare tumor, reference other similar cases and discuss the prognosis of this extremely rare tumor.

\section{Case presentation}

A 42-year-old well-controlled hypothyroid woman presented with a left-sided neck mass that she noted only 2 weeks before. There was no history of previous radiation or family history of thyroid cancer. Serum calcitonin, carcinoembryonic antigen (CEA) and chromogranin A were normal. Neck examination revealed a visible, firm left and isthmic thyroid mass. Ultrasound showed a $4.4 \times 4.1 \times 6.5 \mathrm{~cm}$ heterogeneous mass replacing the left thyroid lobe. The right lobe had typical changes of chronic lymphocytic thyroiditis. A positron emission tomography (PET) scan identified a hypermetabolic mass replacing most of the left lobe and isthmus with a maximum SUV of 17.3 (Fig. 1A). A PET-computed tomography (CT) scan and brain MRI were negative for other primary or metastatic lesions.

\section{Investigation}

A CT-guided core-needle biopsy was performed. The hematoxylin and eosin-stained sections identified a tumor composed of sheets of small-to-medium-sized epithelial cells with $>10$ mitotic figures per $1 \mathrm{~mm}^{2}$. There was no evidence of a papillary thyroid carcinoma. There was stromal material suggesting amyloid, but Congo red and thioflavin $\mathrm{T}$ stains were negative (Fig. 1B). The tumor cells were positive with immunostains for pancytokeratin, synaptophysin (Fig. 1C), neural cell adhesion molecule (CD56) and thyroid transcription factor-1 (Ventana, BenchMark, ULTRA, Tucson, AZ). No reactivity was identified for CK7, CK20, CD45, CD99, ERG (clone EPR3864, done by Quest Diagnostics, Chantilly, VA), chromogranin A, thyroglobulin, calcitonin and CEA. The Ki-67 index was $\sim 90 \%$. A repeat calcitonin immunostain (Autostainer Link 48, Dako; Agilent Technologies, Inc.) and a CGRP immunostain (clone 4901; dilution 1:500; Abcam) were both negative. mRNA in situ hybridization for calcitonin was performed on paraffin sections using a single-strand DNA commercial probe (CAM0017, Histosonda Calcitonin (Cenbimo, Lugo, Spain)), following the manufacturer's protocol, with negative results (Fig. 1D).

\section{Treatment}

A diagnosis of small cell carcinoma of the thyroid was made. The AJCC 8th edition staging was cT3a, cNO and cM0. The case was discussed at an interdepartmental oncology conference with several oncologists and a radiation oncologist and the consensus was to defer surgery and to treat the cancer as a pulmonary small cell carcinoma utilizing aggressive chemotherapy with 4 cycles of cisplatin and etoposide (VP16), followed by consolidative radiation therapy (6600 cGy delivered
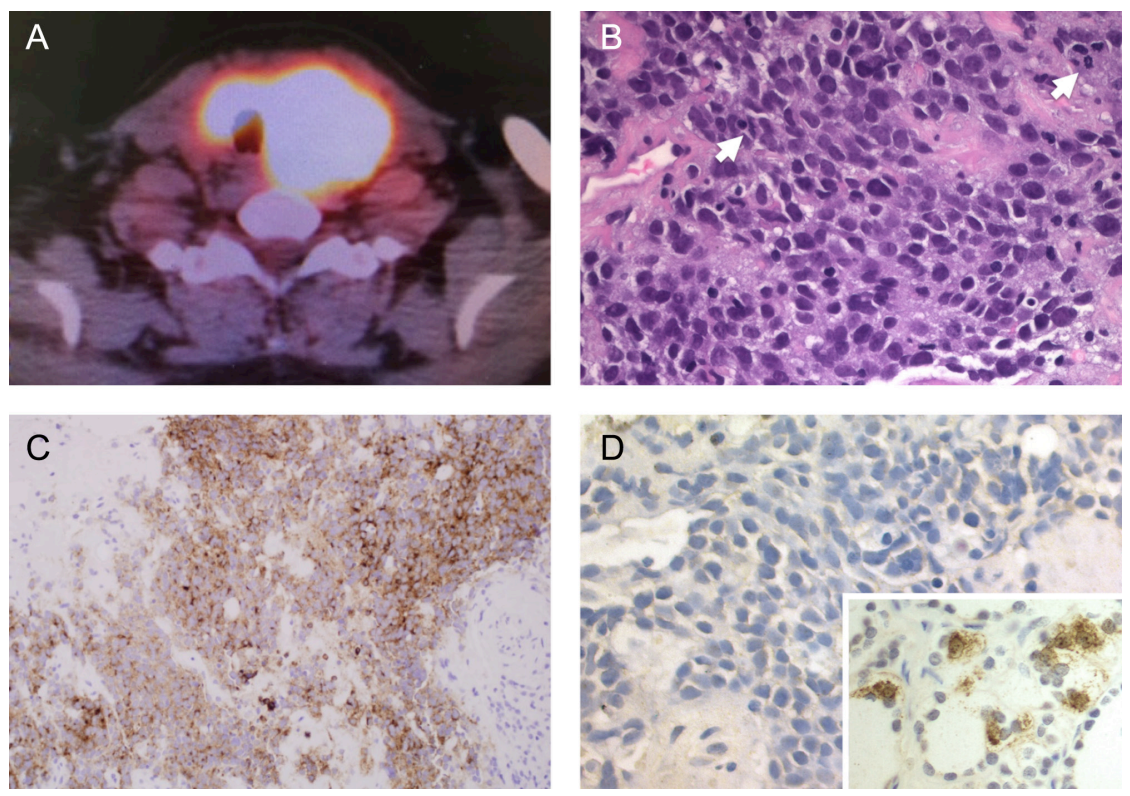

\section{Figure 1}

Calcitonin-negative high-grade neuroendocrine carcinoma of the thyroid gland. There was a hypermetabolic mass in the left lobe and isthmus with a maximum SUV of 17.3 (A, PET scan). There were sheets of small-to-medium sized epithelial cells with identifiable mitotic figures (arrows) and material suggesting amyloid, which was nonreactive with Congo red and thioflavin $T$ stains ( $B, H \& E, 400 x)$. The tumor cells were immunoreactive for synaptophysin (C, 200x). No calcitonin mRNA was detected by in situ hybridization; positivity was found in a case of C-cell hyperplasia used as a positive control (inset) (D, mRNA in situ hybridization, 400x). 
in 33 fractions). Based upon the pulmonary small cell carcinoma data, there would be a $10-15 \%$ chance of cure.

\section{Outcome and follow-up}

There was a complete tumor response to the chemotherapy with negative PET scans at 2 and 6 months and negative neck and chest CT scans. At present, 5 years after treatment, the patient is alive and cancer free. Due to the excellent clinical course, the tumor was reviewed and reclassified as a high-grade neuroendocrine carcinoma (non-small cell type) as it lacked the classic histologic features of a small cell carcinoma.

\section{Discussion}

Despite limited sampling, the findings herein fit well with those of a high-grade neuroendocrine carcinoma. Such tumors are very rare in the thyroid gland and most have been small cell carcinomas (1, 2, 3, 4, 5). Grading of neuroendocrine tumors is organ specific, and the grading of thyroid neuroendocrine carcinomas has not yet been described, but by both the mitotic rate and Ki67 positive rate, this tumor would be a high-grade neuroendocrine carcinoma in any organ.

In the 2017 WHO classification of thyroid tumors, high-grade neuroendocrine carcinomas are not listed as a distinctive type, but they occur and are exceedingly rare. High-grade neuroendocrine carcinomas are divided into two types based upon morphology: small cell carcinoma and non-small cell carcinoma or large cell neuroendocrine carcinoma. In the thyroid, most highgrade neuroendocrine carcinomas have been small cell carcinomas with a poor prognosis. In 1990, Eusebi et al. (1) published two cases of small cell carcinoma. Since that paper, there have been other descriptions of small cell carcinoma of the thyroid $(1,2,3,4,5,6)$. In a large epidemiologic study in England from 1970 to 2004 of extrapulmonary small cell carcinomas (2), 41 of 1618 patients (2.5\%) had a primary thyroid small cell carcinoma. Unfortunately, no clinical follow-up of these tumors was provided. However, in all these other reported primary thyroid small cell carcinoma cases, there was metastatic disease at presentation $(1,3,4,5)$.

Serum calcitonin-negative MTCs have been reported and most are nonsecretory MTCs that do not elevate serum calcitonin, but have occasional tumor cells which are immunoreactive for calcitonin. In this case, two different calcitonin immunostains were negative.
Including this case, there are at least eight reported cases of calcitonin-negative, thyroglobulin-negative, neuroendocrine tumors of the thyroid. There are a similar number of reported thyroglobulin-positive neuroendocrine tumors, and it is unclear whether these tumors should be classified together. These neuroendocrine tumors have had diverse clinical and pathologic findings and many have not been high-grade or the grade was not reported. Sobol et al. (6) presented a case of an 82-yearold woman with a $2 \mathrm{~cm}$ presumed MTC that was negative for calcitonin, CGRP and thyroglobulin, but positive for chromogranin A. Tumor grade was not reported, although it was an aggressive tumor that lacks features of a small cell carcinoma in the photomicrographs. The patient developed metastasis 6 months after resection and died within 2 years. Schmid et al. (7) reported 142 MTCs, one of which lacked calcitonin mRNA and protein expression and was termed an 'atypical' MTC. There was no clinical follow-up. Nakazawa et al. (8) presented a case of a 76-year-old man with a low-grade neuroendocrine carcinoma of the thyroid that was disease free 18 months after surgery. The tumor cells were immunoreactive for CGRP, but not for calcitonin. Liu et al. (9) reported a tumor similar to this case arising in the background of papillary thyroid carcinoma. The tumor cells were calcitonin and thyroglobulin negative, had a Ki67 rate of $40 \%$ and had metastasized to cervical lymph nodes. Ismi et al. (10) reported a 57-year-old man with a highgrade neuroendocrine carcinoma who presented with distant metastases. The tumor had a Ki-67 index of 70\% and was negative for calcitonin and CEA, but stained with chromogranin A and synaptophysin. Kasajima et al. (11) described a $2.8 \mathrm{~cm}$ thyroid nodule in a 48 -year-old-woman that had neuroendocrine differentiation, was negative for calcitonin, but expressed CGRP. There was no reported follow-up. Lastly, Parmer et al. (12) described a 74-yearold woman with a low-grade neuroendocrine tumor that was calcitonin and thyroglobulin negative. Again, there was no long-term follow-up. In these reports, the tumors reported by Sobol and coworkers, Liu and coworkers and Ismi and coworkers have similar high-grade features as in our case. Unlike these cases, however, our patient has not had metastatic disease.

Reported cases of calcitonin-negative neuroendocrine tumors of the thyroid gland are rare, and in particular, there are only a few cases of high-grade neuroendocrine carcinomas. Given the rarity of these tumors, there are no standard guidelines for prognostication and treatment. It has been reported that the $\mathrm{Ki}-67$ proliferation rate prognosticates conventional MTCs (13), and this is likely 
the case with thyroidal neuroendocrine carcinomas, but as these tumors are rare, it has never been studied. It is known that high-grade neuroendocrine tumors of all organs have a poor prognosis. As opposed to small cell carcinomas, which generally present with metastatic disease, a minority of non-small cell (large cell) highgrade neuroendocrine carcinomas present at an earlier stage, and therefore, are potentially curable. This may be more likely in the thyroid gland where a rapidly growing tumor is easily detected and treated.

\section{Declaration of interest}

The authors declare that there is no conflict of interest that could be perceived as prejudicing the impartiality of the research reported.

\section{Funding}

This work was partially supported by grant PI15/01501/co-financedFEDER from the Instituto de Salud Carlos III, Ministry of Economy and Competitiveness, Spain.

Patient consent

Consent for publication of this study was obtained from the patient.

\section{Author contribution statement}

Dr Orrego (endocrinologist) did the biopsy. Drs Chorny and CameselleTeijeiro (pathologists) diagnosed the tumor. All three contributed to writing the manuscript.

\section{References}

1 Eusebi V, Damiani S, Riva C, Lloyd RV \& Capella C. Calcitonin free oat-cell carcinoma of the thyroid gland. Virchows Archiv A Pathological Anatomy and Histopathology 1990417 267-271. (https:// doi.org/10.1007/BF01600144)

2 Wong YNS, Jack RH, Mak V, Henrik M \& Davies EA. The epidemiology and survival of extrapulmonary small cell carcinoma in South East England, 1970-2004. BMC Cancer 20099 209-215 (https://doi.org/10.1186/1471-2407-9-209)

3 Beach DF, Klump WJ, Haddad G, Reid LM, Schwarting R \& Hageboutros A. Extrapulmonary small cell: a novel case of small cell carcinoma of the thyroid gland. Medical Oncology 201229 1405-1408. (https://doi.org/10.1007/s12032-011-9996-7)

4 Mussazhanova Z, Miura S, Stanojevic B, Rougounovitch T, Saenko V, Shiraishi T, Kurashige T, Shichijo K, Kaneko K, Takahashi H, et al. Radiation-associated small cell neuroendocrine carcinoma of the thyroid: a case report with molecular analyses. Thyroid $20142 \mathbf{2 4}$ 593-598. (https://doi.org/10.1089/thy.2013.0214)

5 Ortansa MR, Rodica A, Dumitrache M, Adriana C, Oprea V \& Mitulescu D. Small cell neuroendocrine thyroid carcinoma. Therapeutics, Pharmacology and Clinical Toxicology 201418 171-173.

6 Sobol RE, Memoli V \& Deftos LJ. Hormone-negative, chromogranin A-positive endocrine tumors. New England Journal of Medicine 1989 320 444-447. (https://doi.org/10.1056/NEJM198902163200707)

7 Schmid KW \& Ensinger C. "Atypical" medullary thyroid carcinoma with little or no calcitonin expression. Virchows Archiv 1998433 209-215. (https://doi.org/10.1007/s004280050238)

8 Nakazawa T, Cameselle-Teijeiro J, Vinagre J, Soares P, Rousseau E, Eloy C \& Sobrinho-Simões M. C-cell-derived calcitonin-free neuroendocrine carcinoma of the thyroid: the diagnostic importance of CGRP immunoreactivity. International Journal of Surgical Pathology 201422 530-535. (https://doi.org/10.1177/1066896914525228)

9 Liu Z, Jin M, Su C, Ren J, Wan F, Guan Q, Miao Z, Chen G \& Wang G. Thyroid neuroendocrine cancer accompanied with multiple papillary thyroid carcinomas: a case report. International Journal of Clinical and Experimental Pathology $201692396-2401$.

10 Ismi O, Arpaci RB, Berkesoglu M, Dag A, Sezer E, Koray KB \& Vayisoğlu Y. Calcitonin-negative neuroendocrine tumor of thyroid gland mimicking anaplastic carcinoma: an unusual entity. Gland Surgery 20154 344-349. (https://doi.org/10.3978/j.issn.2227684X.2015.01.02)

11 Kasajima A, Cameselle-Teijeiro J, Loidi L, Takahashi Y, Nakashima N, Sato S, Fujishima F, Watanabe M, Nakazawa T, Naganuma $\mathrm{H}_{\text {, }}$ et al. A calcitonin non-producing neuroendocrine tumor of the thyroid gland. Endocrine Pathology 201627 325-331. (https://doi. org/10.1007/s12022-016-9416-9)

12 Parmer M, Milan S \& Torabi A. Calcitonin-negative neuroendocrine tumor of the thyroid: follicular or parafollicular cell of origin? International Journal of Surgical Pathology 201725 191-194. (https:// doi.org/10.1177/1066896916670989)

13 Frank-Raue K, Machens A, Leidig-Bruckner G, Rondot S, Haag C, Schulze E, Lorenz A, Kreissl MC, Dralle H, Raue F, et al. Prevalence and clinical spectrum of nonsecretory medullary thyroid carcinoma in a series of 839 patients with sporadic medullary thyroid carcinoma. Thyroid 201323 294-300. (https://doi.org/10.1089/ thy.2012.0236)

Received in final form 12 March 2018

Accepted 27 March 2018 